\title{
Gravitational lensing of gravitational waves: wave nature and prospects for detection
}

\author{
Ashish Kumar Meena, ${ }^{1 \star}$ Jasjeet Singh Bagla, ${ }^{1 \dagger}$ \\ ${ }^{1}$ Indian Institute of Science Education and Research Mohali, Knowledge City, Sector 81, Sahibzada Ajit Singh Nagar, Punjab 140306, India
}

13 December 2019

\begin{abstract}
We discuss the gravitational lensing of gravitational wave signals from coalescing binaries. We delineate the regime where wave effects are significant from the regime where geometric limit can be used. Further, we focus on the effect of micro-lensing and the combined effect of strong lensing and micro-lensing. We find that micro-lensing combined with strong lensing can introduce time varying phase shift in the signal and hence can lead to detectable differences in the signal observed for different images produced by strong lensing. This, coupled with the coarse localization of signal source in the sky for gravitational wave detections, can make it difficult to identify the common origin of signal corresponding to different images and use observables like time delay. In case we can reliably identify corresponding images, micro-lensing of individual images can be used as a tool to constrain properties of micro-lenses. Sources of gravitational waves can undergo microlensing due to lenses in the disk/halo of the Galaxy, or due to lenses in an intervening galaxy even in absence of strong lensing. In general the probability for this is small with one exception: Extragalactic sources of gravitational waves that lie in the galactic plane are highly likely to be micro-lensed. Wave effects are extremely important for such cases. In case of detections of such sources with low SNR, the uncertainty of occurrence of microlensing or otherwise introduces an additional uncertainty in the parameters of the source.
\end{abstract}

Key words: gravitational lensing: strong - gravitational lensing: micro - gravitational waves

\section{INTRODUCTION}

The recent detection of gravitational wave (GW) signal (The LIGO Scientific Collaboration \& the Virgo Collaboration 2018) from coalescing binaries opens up a new window to observe the Universe (Rosswog 2015; Wei \& Wu 2017). The upcoming runs of LIGO and Virgo with increased sensitivity and new detector facilities (KAGRA Collaboration 2018) will increase the number of observed GW signals significantly. As a result, the possibility of detecting a gravitationally lensed GW will also increase.

Propagation of gravitational waves is influenced by gravitational fields and results in a deflection in a manner similar to electromagnetic waves (Lawrence 1971; Ohanian 1974). In the case of electromagnetic radiation, geometric optics is sufficient to study the effects of gravitational lensing as the sources have a finite size and the wavelength of the radiation is much smaller than all other scales of interest (Deguchi \& Watson 1986; Schneider et al. 1992; Matsunaga

\footnotetext{
^ E-mail: ashishmeena@iisermohali.ac.in

$\dagger$ E-mail: jasjeet@iisermohali.ac.in
}

\& Yamamoto 2006). Sources of gravitational waves are fairly compact and the wavelength of radiation that can be detected by existing and future detectors is larger than the region of emission. The wavelength of gravitational waves is comparable with the Schwarzschild radius of many astronomical objects. Thus in the case of gravitational waves, the geometric optics is not always valid (Bontz \& Haugan 1981; Deguchi \& Watson 1986; Nakamura 1998; Baraldo et al. 1999; Christian, Vitale \& Loeb 2018). In LIGO frequency band $(10 \mathrm{~Hz}-10 \mathrm{kHz})$ for galaxy mass lenses geometric optics is sufficient (Varvella et al. 2004) as the wavelengths are much smaller than the size of a galaxy. Under geometric optics approximation, for galaxy mass lenses, the GW signal gets multiply imaged, and each signal is amplified by a constant factor. Although extra amplification can help us in observing events that are beyond LIGO range, the amplification factor and luminosity distance to the source have a degeneracy that can introduce errors in the analysis (Broadhurst et al. 2018, 2019). If we can identify the multiple images in a lensed system then the time delay between these different signals can constrain the cosmological parameters (Sereno et al. 2011). The presence of small compact objects 
(micro-lenses) in the lens can further affect the signal. In this case the wave nature can become important and hence the effect of lensing is a combination of a wavelength dependent amplification factor as well as the phase of the signal (Diego et al. 2019).

In this work we discuss the effects of micro-lensing in strongly lensed GW signal. The effects of micro-lensing are non-negligible in the LIGO frequency band (Christian, Vitale \& Loeb 2018; Diego et al. 2019). As we shall see, these effects introduce frequency dependence in amplification as well as the phase of the lensed GW signal.

In section $\S 2$, we review the basics of wave optics in gravitational lensing. Results are given in §3. Summary and conclusions are given in $\S 4$. We also discuss possibilities for future work in this section.

\section{BASICS OF GRAVITATIONAL LENSING}

In this section, we review some aspects of wave effects in gravitational lensing, which is relevant for our analysis (Schneider et al. 1992; Nakamura \& Deguchi 1999; Takahashi \& Nakamura 2003). To describe the effect of the gravitational lens, one can consider a perturbed FRW as background metric, given by

$d s^{2}=-(1+2 U) d t^{2}+a^{2}(1-2 U) d \mathbf{r}^{2}=g_{\mu \nu}^{(B)} d x^{\mu} d x^{\nu}$,

where $U$ is the gravitational potential of the lens. The gravitational waves in this background are described as a tensor perturbation, $h_{\mu v}$. The linear perturbation can be written as $\phi e_{\mu \nu}$; where $\phi$ represents the amplitude, and $e_{\mu \nu}$ is the polarization tensor of the gravitational wave. During propagation, the change in polarization tensor due to the presence of the lens is negligible (Takahashi \& Nakamura 2003; Misner et al. 1973). As a result, one can assume the polarization vector to be a constant. The propagation equation (to the leading order) for the scalar amplitude $\phi$, in frequency domain is,

$$
\left(\nabla^{2}+\widetilde{\omega}^{2}\right) \widetilde{\phi}=4 \widetilde{\omega}^{2} U \widetilde{\phi}
$$

where $\widetilde{\omega}=2 \pi f$, and $f$ is the frequency of the gravitational wave. One can solve the equation (2) using Kirchhoff diffraction integral (Baraldo et al. 1999; Takahashi \& Nakamura 2003) for waves coming from a source at a distance $D_{S}$ to the observer. Following Takahashi \& Nakamura (2003), the amplification factor, $F(f)$ is defined as the ratio of lensed and unlensed $(U=0)$ gravitational wave amplitudes $\widetilde{\phi}$. This implies that in no-lens limit $(U=0)$, the amplification factor is unity $(|F|=1)$.

In order to calculate the amplification factor, we need to solve the equation (2) for a given lens system. Under thin lens approximation, for a gravitational wave source at a distance $D_{S}$ and a lens at $D_{L}$, the amplification factor at the observer is given by Takahashi \& Nakamura (2003),

$F(f, \mathbf{y})=\frac{D_{S} \xi_{0}^{2}\left(1+z_{L}\right)}{c D_{L} D_{L S}} \frac{f}{i} \int d^{2} \mathbf{x} \exp \left[2 \pi i f t_{d}(\mathbf{x}, \mathbf{y})\right]$,

where $\mathbf{x}=\boldsymbol{\xi} / \xi_{0}, \mathbf{y}=\boldsymbol{\eta} D_{L} / \xi_{0} D_{S}$ is the dimensionless source position in the source plane, $\xi_{0}$ is an arbitrary length scale, $z_{L}$ is the lens redshift and $t_{d}$ is the arrival time, given by

$t_{d}(\mathbf{x}, \mathbf{y})=\frac{D_{S} \xi_{0}^{2}\left(1+z_{L}\right)}{c D_{L S} D_{S}}\left[\frac{1}{2}|\mathbf{x}-\mathbf{y}|^{2}-\psi(\mathbf{x})+\phi_{m}(\mathbf{y})\right]$.
The constant $\phi_{m}(\mathbf{y})$ does not depend on the lens properties and one can choose a form to simplify calculations. Here we choose $\phi_{m}(\mathbf{y})$ such that the value of time delay for minima image (image for which time delay is a global minimum) is zero: time delays for other points are measured with respect to this image. The phase of the amplification factor can be defined as, $\Delta \phi=-i \ln [F /|F|]$.

In the limit of geometric optics approximation $(f \gg$ $t_{d}^{-1}$ ), the integral in equation (3) is a highly oscillatory function, and the amplification factor gets a significant contribution only from stationary points of the integrand. The form of amplification factor in geometric optics limit is

$F(f, \mathbf{y})=\sum_{i} \sqrt{\left|\mu_{j}\right|} \exp \left(2 \pi i f t_{d, j}-i \pi n_{j}\right)$,

where $t_{d, j}$ is the value of time delay for $j$-th image, $\mu_{j}$ is the amplification of the $j$-th image and $n_{j}$ is the Morse index with values $0,1 / 2,1$ for images corresponding to minima, saddle and maxima of the time delay function, respectively (Takahashi \& Nakamura 2003; Blandford \& Narayan 1986). One can see that the lensed gravitational waveform corresponding to maxima (saddle) of the time delay has a phase difference of $e^{-i \pi}\left(e^{\frac{-i \pi}{2}}\right)$ compared to the waveform corresponding to the minima of time delay (Dai \& Venumadhav 2017).

In order to find out the amplification factor for different lens models, we solve equation (3) numerically. However, for a point mass lens, the solution of equation (3) can be obtained analytically and is given in terms of the hypergeometric function,

$$
\begin{aligned}
F(\omega, y)= & \exp \left[\frac{\pi \omega}{4}+\frac{i \omega}{2}\left\{\ln \left(\frac{\omega}{2}\right)-2 \phi_{m}\left(x_{m}\right)\right\}\right] \\
& \Gamma\left(1-\frac{i \omega}{2}\right){ }_{1} F_{1}\left(\frac{i \omega}{2}, 1 ; \frac{i \omega}{2} y^{2}\right),
\end{aligned}
$$

where $\omega=8 \pi G\left(1+z_{L}\right) M_{L} f / c^{3}, \phi_{m}(\mathbf{y})=\left(x_{m}-y\right)^{2} / 2-\ln x_{m}$ with $x_{m}=\left(y+\sqrt{y^{2}+4}\right) / 2$. Here we used Einstein radius as the scale length $\xi_{0}=\left(4 G M_{L} D_{L} D_{L S} / c^{2} D_{S}\right)^{1 / 2}$. Using geometric optics approximation $(\omega \gg 1)$, the above equation reduces to,

$F(\omega, y)=\sqrt{\left|\mu_{+}\right|}-i \sqrt{\left|\mu_{-}\right|} \exp \left[2 \pi i f \Delta t_{d}\right]$,

where $\mu_{ \pm}$, are the amplification factor for primary and secondary images for a point mass lens in geometric optics limit and $\Delta t_{d}$ is the time delay between these two images. One can see the oscillatory behavior of the amplification factor at higher frequencies. To get the conventional expression for amplification factor, we need to take a square followed by an average of equation (7). In our approach we have assumed a point source for gravitational waves. A further refinement where we take the finite size of the emitting region can be made (Matsunaga \& Yamamoto 2006).

\section{RESULTS}

In this section, we present the results of our study of gravitational lensing of gravitational waves emitted from a coalescing binary. For illustration we neglect the role of eccentricity of the orbit. This section is further divided into subsections, 


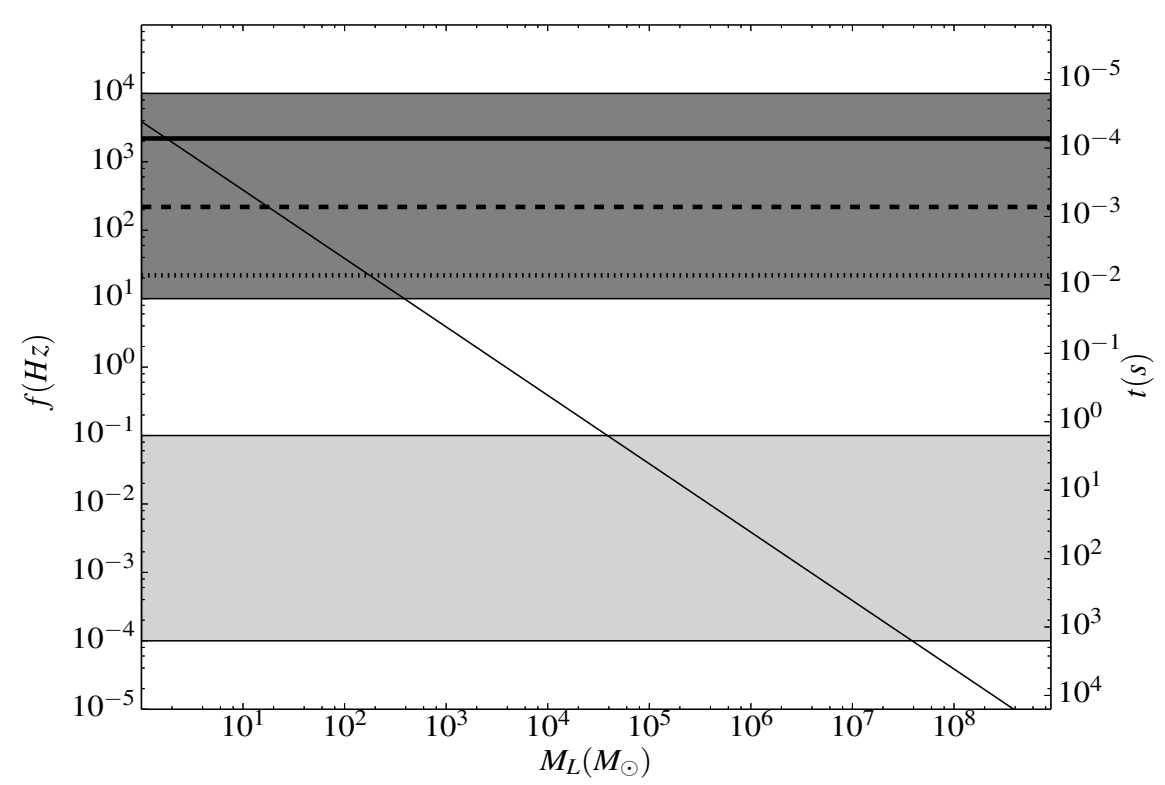

Figure 1. Lens mass versus gravitational wave frequency: The diagonal thin line from top left to bottom right represents the critical frequency below which wave effects are essential to calculate the lensing effects for a given point mass. The light and dark shaded regions represent the relevant frequencies for LISA and LIGO, respectively. The horizontal lines (dotted, dashed, thick solid line) represents the GW frequency at the innermost stable circular orbit (ISCO) emitted by the source binaries of masses, $100 M_{\odot}+100 M_{\odot}, 10 M_{\odot}+10 M_{\odot}$, $1 M_{\odot}+1 M_{\odot}$, respectively.

considering different circumstances in which a gravitational wave can be affected by a gravitational lens.

\subsection{Applicability of geometrical optics}

In case of lensing of electromagnetic radiation, due to the small wavelength, use of geometric optics is adequate as corrections from the wave optics are negligible (Schneider et al. 1992). However, the wavelength of gravitational waves that can be detected with existing and future detectors is much larger and is comparable to the relevant scales of some gravitational lenses. Therefore in order to discuss the lensing of gravitational waves, we need to ascertain whether wave effects are significant or not? We find that for wavelengths equal to or larger than the path difference between the multiple images of the source, one should use wave optics instead of geometric optics. Taking equation (3) into account, one can write the above condition in mathematical form as (Takahashi 2017),

$2 \pi f t_{d} \lesssim 1$

where $f$ is the frequency of the gravitational waves and $t_{d}$ is the time delay between the images. Figure (1) shows the plot of the equation (8) for a point mass lens. The thin solid line denotes the condition $2 \pi f t_{d}=1$. Below this line one should use the wave optics results as the wavelength of the gravitational waves is much larger than the path difference between the two images, which is of the order of the Schwarzschild radius of the lens. Above but near this line, the correction terms from diffraction effects continue to be non-negligible. As one moves away from this line, the correction terms due to diffraction effects become negligible, and finally one can use geometric optics if the wavelength of the gravitational wave is much smaller than the Schwarzschild radius of the lens. Geometric optics is a good approximation if we are a factor of at least a few above this line.

The light and dark shaded regions represent the frequency ranges covered by LISA and LIGO, respectively. The horizontal lines (dotted, dashed, thick solid line) represents the frequency of the gravitational waves at the innermost stable circular orbit (ISCO) emitted by the source binaries of masses, $100 M_{\odot}+100 M_{\odot}, 10 M_{\odot}+10 M_{\odot}, 1 M_{\odot}+1 M_{\odot}$, respectively. For the purpose of illustration we have neglected the effects of eccentricity and post-Newtonian corrections while computing the relevant frequency. The corresponding time delay is shown in the right side of the plot. One can see that for most of the relevant sources, the frequency of gravitational waves at ISCO lies in the LIGO band and the wave effects are only important in case of a micro-lensing scenario (Christian, Vitale \& Loeb 2018).

The frequency dependent amplification and phase shift leads to chromatic effects and this is the key signature of wave effects. These effects modify the waveform from coalescing binaries as the frequency keeps on increasing with time in the signal. The changes can be large enough to lead to mis-identification of source properties. Christian, Vitale \& Loeb (2018) have shown that disentangling the effects of micro-lensing is feasible for detections with a high signal to noise ratio $(S N R \geq 30)$.

\subsection{Micro-lensing Effects}

The possibility of strong lensing of gravitational waves gives us the opportunity to observe the same source more than once due to the nonzero time delays (Piórkowska et al. 2013; Smith et al. 2018; Li et al. 2018; Haris et al. 2018; Hannuksela et al. 2019). In general, these different signal have different constant amplification factor, depending on the ge- 

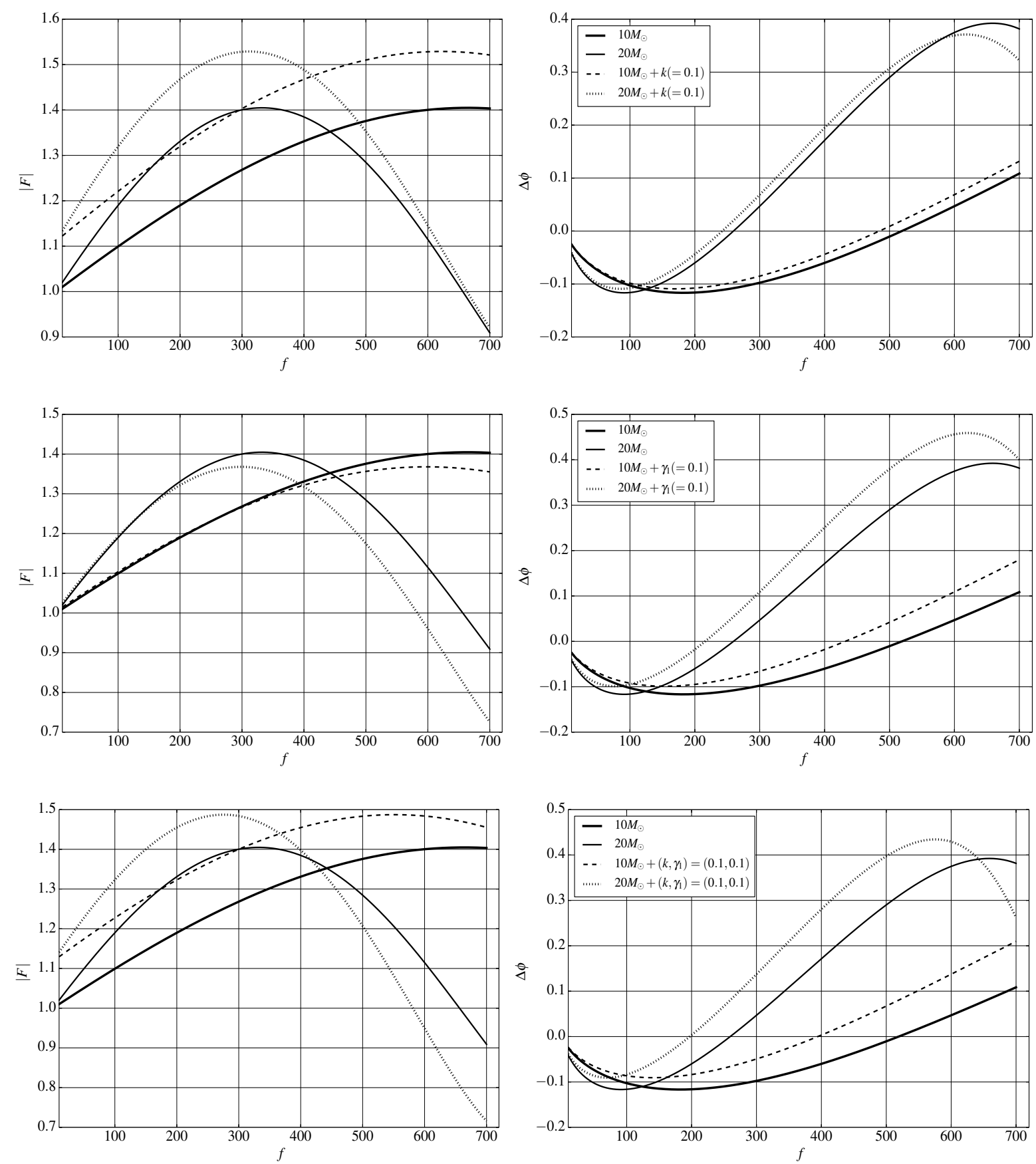

Figure 2. Micro-lensing within a strongly lensed image: The left column represents the amplification factor for two different micro-lenses within a strong lens represented by external shear and convergence. The right column shows the corresponding phase. For each row, the mass of the micro-lens and the values for external convergence and shear are mentioned in the right side of the respective row. The $\mathrm{x}$-axis represents the frequency of gravitational waves in Hertz. The y-axis represents the amplification factor $(|F|)$ and phase $(\Delta \phi)$ in left and right panel, respectively.

ometry of the lensing system. However, as we know smaller mass compact objects (stars, stellar remnants, black holes) are a part of galaxies and can further affect the gravitational wave signal via micro-lensing. The lensing due to these micro-lenses can also help us in observing intermediate mass black holes (IMBH) (Lai et al. 2018). As mentioned above, in LIGO frequency range, only micro-lensing $\left(M_{\text {lens }} \leq 10^{3} \mathrm{M}_{\odot}\right)$ can give rise to the significant frequency dependent effects.
For simplicity we only consider a single object as a potential micro-lens. In order to calculate the effect of microlensing, we choose the object to be located at the origin. The effect of the galaxy as a whole does not vary significantly over the relevant length scale of a star. As a result, the effect of the galaxy can be describe in terms of constant external convergence $\left(\kappa^{\prime}\right)$ and constant shear $\left(\gamma_{1}^{\prime}, \gamma_{2}^{\prime}\right)$. Due to the presence of external effects, the resultant lensing 

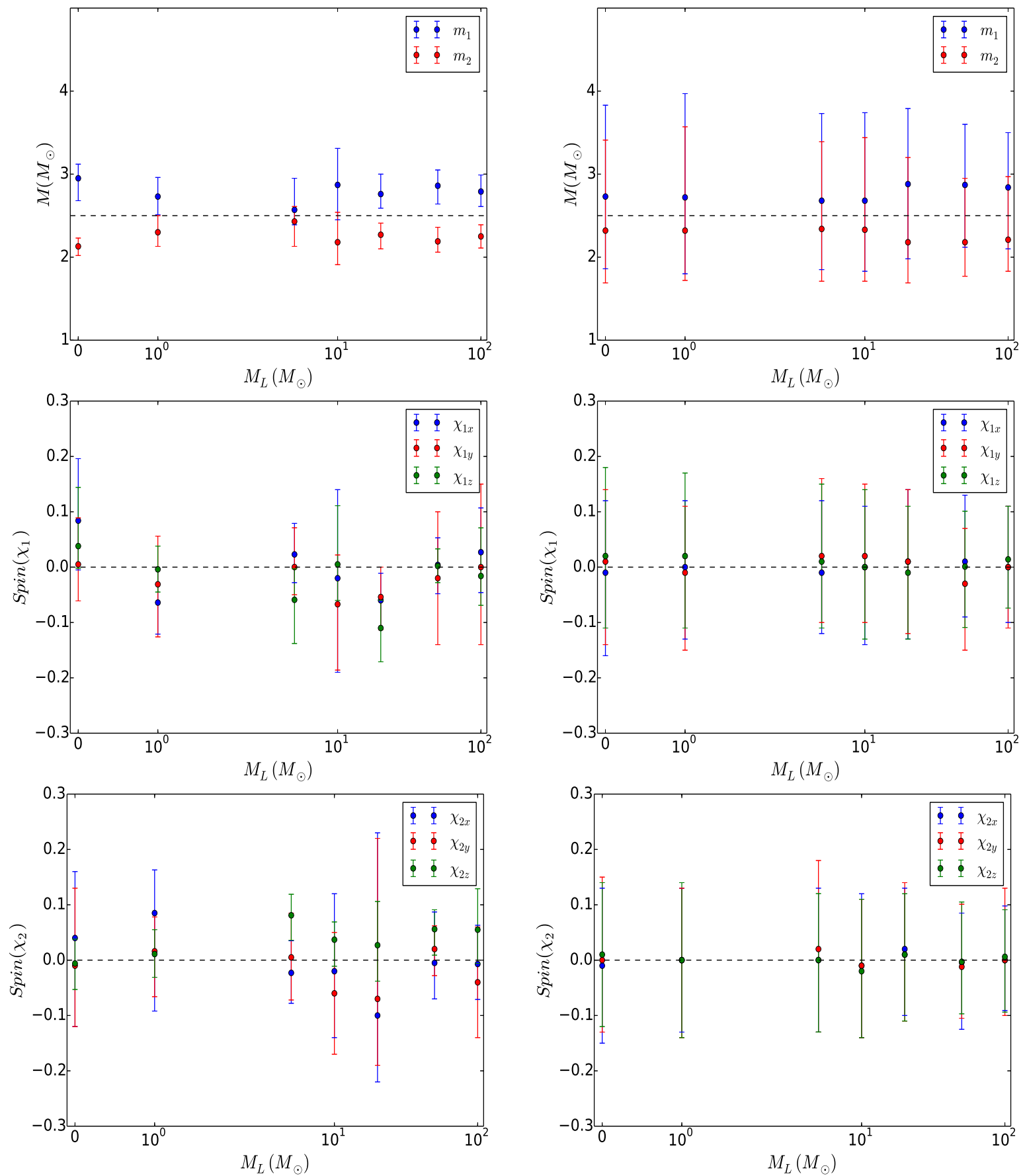

Figure 3. Parameter estimation of a lensed GW signal at high and small SNR values: The left column shows the parameter estimation for a GW signal at SNR value 34 (in unlensed case), whereas the right column shows the parameter estimation at SNR value 10 (in unlensed case). The $\mathrm{x}$-axis represents the microlens mass. The y-axis represents the different parameter values. In different panels, the horizontal dotted line represents the input value of the corresponding parameters. The top row represents the mass estimation of binary components for different microlens values. The middle and bottom row represents the spin component estimation for first and second binary components, respectively. The error bars show one sigma error in the estimation of different parameters.

potential of the star will be modified and can be written as (Schneider et al. 1992):

$$
\begin{aligned}
\psi\left(x_{1}, x_{2}\right)= & \ln \left(\sqrt{x_{1}^{2}+x_{2}^{2}}\right)+\frac{\kappa^{\prime}}{2}\left(x_{1}^{2}+x_{2}^{2}\right) \\
& +\frac{\gamma_{1}^{\prime}}{2}\left(x_{1}^{2}-x_{2}^{2}\right)+\gamma_{2}^{\prime} x_{1} x_{2} .
\end{aligned}
$$

Using equation (3) and (9), we can calculate the effect of the star on the amplification factor as well as the phase of the gravitational wave signal.

Here we show results for three different possibilities: non-zero convergence, non-zero shear, and non-zero convergence and shear for a lens at redshift $z=0.05$ and the source positioned at $\left(y_{1}, y_{2}\right)=(1,0)$ at redshift $z=0.2$. The mass of 

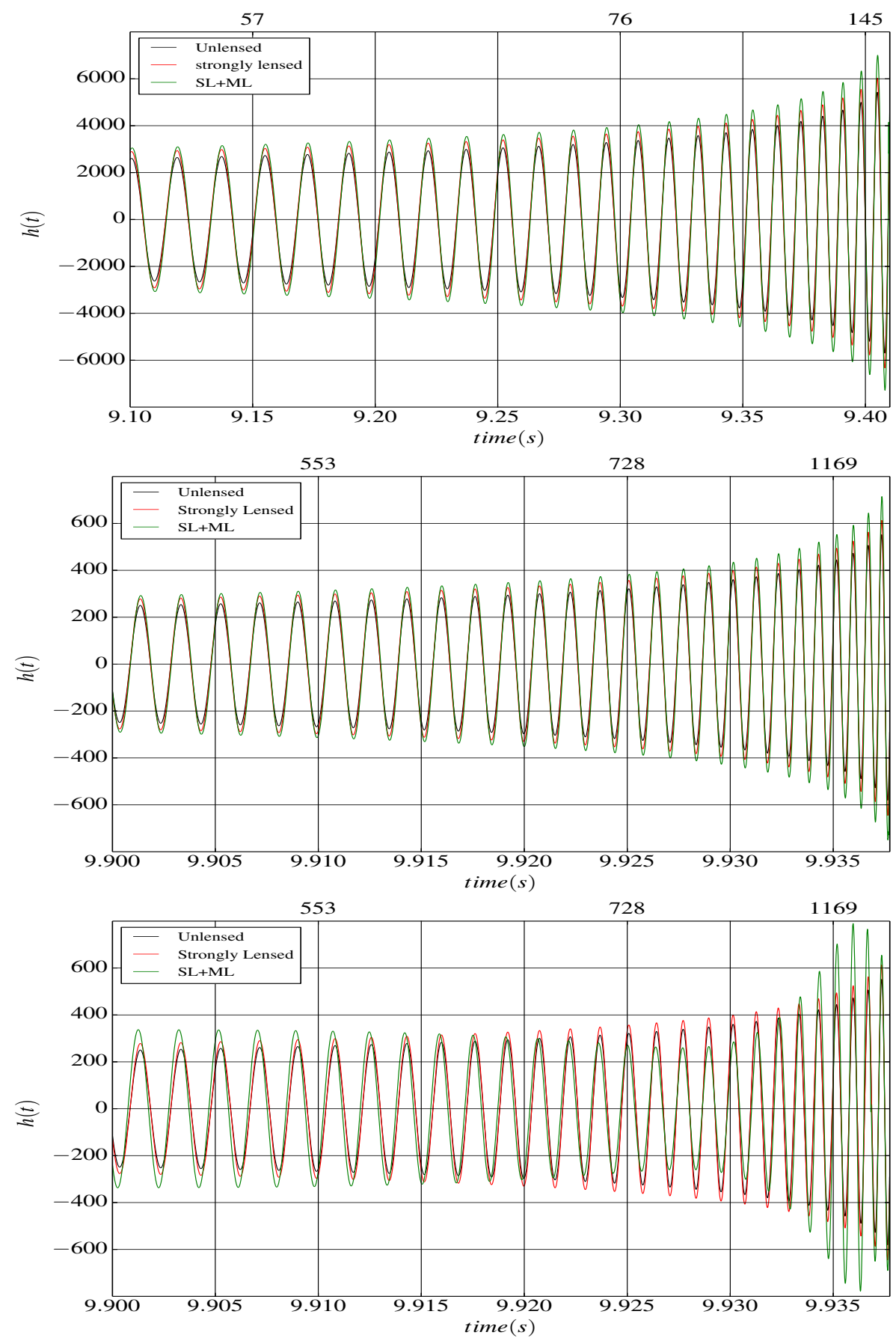

Figure 4. Effect of lensing on gravitational waveform in time domain. The top panel is for a $10 \mathrm{M}_{\odot}+10 \mathrm{M}_{\odot}$ binary, the lower panels are for a $1 \mathrm{M}_{\odot}+1 \mathrm{M}_{\odot}$ binary. The black curve shows unlensed signal, the red curve shows the effect of strong lensing (in the geometric limit), and the green curve shows the effect of micro-lensing in addition to strong lensing. The top panel includes micro-lensing by a $10 \mathrm{M}_{\odot}$ lens, the middle panel includes micro-lensing by a $1 \mathrm{M}_{\odot}$ lens, and the lowest panel includes micro-lensing by a $20 \mathrm{M}_{\odot}$ lens. In each panel the $\mathrm{x}$-axis shows the time, the corresponding frequency of gravitational waves is marked on the top of each panel. Each panel shows the gravitational wave amplitude as a function of time (and frequency). The unlensed signal is amplified uniformly by strong lensing, independent of frequency. However the inclusion of micro-lensing brings in the frequency dependence in the amplification where the amplitude of the signal gets amplified more at some frequencies. This can be seen in all the rows but it is seen most dramatically in the lowest row where this introduces a strong modulation. Such modulation is seen if we cover a wider range of frequencies, i.e., if the signal is from a lower mass source, or if the micro-lens is much more massive than a sun like star. There is also a frequency dependent phase shift that is obvious from lowest panel and can be read off in right panels of Figure 2. 
the micro-lens is fixed to $10 M_{\odot}$ and $20 M_{\odot}$. As one can see from figure (2), the non-zero value of convergence (shear) introduces a frequency dependence in the amplification factor as well as in the phase (these would have been independent of frequency in the absence of micro-lensing). Further, this frequency dependence is highly sensitive to the lens parameters $\left(M, \kappa, \gamma_{1}, \gamma_{2}\right)$. For example, if we change the mass on the micro-lens from $10 M_{\odot}$ to $20 M_{\odot}$, the amplification and phase pattern show significant changes. Similarly, if we consider a different combination of $\left(\kappa, \gamma_{1}, \gamma_{2}\right)$ which is equivalent to shifting the position of the micro-lens, the amplification pattern changes (see figure 2). The effect on the chirp signal from coalescing binaries is to modulate the amplitude as we have a frequency dependent amplification, and also to introduce time varying phase shifts.

The effect of these oscillations on parameter estimation is shown in figure (3). We have considered the effect of the microlens and ignored the effect of the strong lens galaxy as that does not introduce any frequency dependent variations in the signal. The left and the right column represents the parameter estimation of different binary parameters for a $2.5 \mathrm{M}_{\odot}+2.5 \mathrm{M}_{\odot}$ binary for different SNR values. The SNR value in the left column is 34 for the unlensed signal, whereas the SNR value in the right column is 10 for the unlensed signal. These cover a reasonable range in SNR and give us a glimpse of what to expect. Here we used PyCBC for parameter estimation (Biwer, et al. 2019). As one can see in the right column of figure (3), at small SNR values $(\sim 10)$, the estimated values of various binary parameters do not differ significantly within one sigma errors. However, if the SNR value is larger than 30, then the microlensing effects on the parameter estimation can be distinguished within one sigma errors. Hence, the effect of microlensing is to modify the estimated values of binary parameters. This can be seen in the middle and bottom panel of the left column of figure (3), where a non-zero spin is estimated even though the input spin is zero. It is interesting to note that the extent of the allowed region remains similar to the case of no microlensing (marked by 0 on the x-axis) but the allowed region moves around with variation in the microlens mass. These modifications can be more significant if we also take into account the impact of the host galaxy as the effect of the host galaxy can further increase the SNR value(figure 2).

This introduces a severe problem in identifying different counterparts of a strongly lensed GW signal as the microlensing can introduce a different type of frequency dependence in different images.

Keeping source properties the same, if we explore the effects with changes in the mass of the micro-lens, we find that the form of variation of amplification and phase shift with frequency is monotonic in the LIGO range for micro-lenses with a smaller mass $\left(\sim \mathrm{M}_{\odot}\right)$, whereas it undergoes oscillations for a larger mass $\left(>10^{2} \mathrm{M}_{\odot}\right)$. This is to be expected as with larger masses we gradually approach the geometric limit.

For better visualization, figure (4) represents the lensed waveform in the time domain. We show the expected signal in the final moments before coalescence for three cases. We see here that for the low mass binary we can probe gravitational waves of a higher frequency and hence we are able to see the effect of oscillation in amplification. No such feature is seen in the signal from the higher mass binary as it does not reach higher frequencies. However, a frequency dependent amplification is seen in all cases. There is a frequency dependent phase shift that is not obvious from these figures but which is large enough to mislead in the mapping from the signal to the source parameters. As micro-lensing does not affect each sight line in the same manner, a comparison of the red and the green color waveform in bottom panel of figure (4) is a representation of the differences that we may encounter due to this effect. It is clear from here that it may be difficult to identify signal from different strongly lensed images as coming from the same source due to the variations introduced by micro-lensing.

The probability of a micro-lensing event depends on many factors but it is not small. We are doing a detailed estimation but a preliminary analysis suggests that a few percent of the images are likely to be affected by microlensing. This order of magnitude analysis is done for an elliptical galaxy acting as a gravitational lens lens with de Vaucouleurs profile along with the Salpeter initial mass function. The average microlensing event rate for such a galaxy lens is of the order of $10^{-2}$. If we specifically consider the microlensing near half-light radius, then the value of optical depth is larger than the average value: this is perhaps more relevant as the impact parameter in most cases is of this order or smaller. Similarly, if we consider microlensing due to an edge-on spiral galaxy, then the probability of microlensing can further rise as the projected density of the microlenses within the disk is much higher. On the other hand, the probability for microlensing for off plane images is much lower in this case.

\section{CONCLUSIONS}

We have described the effects of micro-lensing alone and in case of a strongly lensed GW signal. As shown above, micro-lensing is the only way in which lensed signal in LIGO frequency band can get frequency dependent effects. The micro-lens may reside in our galaxy or it may be embedded in a bigger lens, e.g., an intervening galaxy. The effects of micro-lensing depend strongly on the lens parameters. For large values of SNR (>30), as we change any of the lens parameters, the variation of amplification and phase with frequency changes significantly. Because of the possibility that different counterparts of strongly lensed GW signal can get affected differently by micro-lensing, or only one image may be affected significantly, it introduces a real challenge in identifying the counterparts of the strongly lensed GW signal. The other difficulty in identifying the different counterparts of a strongly lensed GW signal is the poor localization of the GW signal. However, this identification can be possible if SNR is large enough (more than about 30) but most detections fall below this threshold. Apart from that, if the magnification factor due to the strong lens is large, then the possibility of microlensing is unavoidable. In such a case, even a few solar mass microlens can introduce significant distortions in the observed GW signal (Diego et al. 2019).

The challenge can be turned into an opportunity if a counterpart is observed in the electromagnetic radiation as this may permit better localization and identification of the images. In such cases we can recover information about the 
wave effects and parameters of the micro-lens. Here, the sensitivity of the amplification and phase difference to parameters of the lens are useful features. In addition, time delay measurements with optical counterparts can be used to infer a lot of information about the lens and cosmological parameters (Piórkowska et al. 2013; Hongsheng \& Xilong 2018).

Gravitational lensing in the LISA band by much heavier lenses retains effects of the wave nature and hence it should be possible to detect these (Liao, Biesiada \& Fan 2019). However, lens masses required are in a range where few potential lens candidates are available. We propose that super massive black holes in intervening galaxies may produce effects that can be tracked in the LISA band. Observations here are sensitive to the mergers of very massive black holes, or to other coalescing binaries at epochs much before the final merger. In the latter case, superposition of signals with different time delays can produce modulation effects (Takahashi \& Nakamura 2003; Sereno et al. 2010).

Micro-lensing can affect gravitational waves from distant sources even in the absence of strong lensing. This may happen due to a lens in an intervening galaxy or a lens in the Galaxy. The probability for this to happen is small in most directions but is relatively (Wood \& Mao 2005) very high in the galactic plane region. Given that this can affect signal from sources in more than $10 \%$ of the sky with a high probability, effects of micro-lensing need to be taken into account seriously. It has been shown (Christian, Vitale \& Loeb 2018) that for detections with a high SNR it is possible to disentangle the micro-lensing effects and source parameter determination. However, it is important that for other events we budget for the uncertainty that is introduced because of the likelihood of micro-lensing.

\section{ACKNOWLEDGEMENTS}

AKM would like to thank CSIR for financial support through research fellowship No.524007. We thank the anonymous referee for useful comments. We acknowledge the use of IISER Mohali HPC facility. This research has made use of NASA's Astrophysics Data System Bibliographic Services.

\section{REFERENCES}

Baraldo C., Hosoya A., Nakamura T. T., 1999, Phys. Rev. D, 59, 083001

Biwer C. M., Capano C. D., De S., Cabero M., Brown D. A., Nitz A. H., Raymond V., 2019, PASP, 131, 024503

Blandford R., Narayan R., 1986, ApJ, 310, 568

Bontz R. J., Haugan M. P., 1981, Astrophysics and Space Science, 78,199

Broadhurst T., Diego J. M., Smoot George I., 2018, arXiv eprints, p. arXiv:1802.05273

Broadhurst T., Diego J. M., Smoot George F. I., 2019, arXiv eprints, p. arXiv:1901.03190

Christian P., Vitale S., Loeb A., 2018, PhRvD, 98, 103022

Dai L., Venumadhav T., 2017, arXiv e-prints, p. arXiv:1702.04724

Deguchi S., Watson W. D., 1986, The Astrophysical Journal, 307, 30

Deguchi S., Watson W. D., 1986, PhRvD, 34, 1708

Diego J. M., Hannuksela O. A., Kelly P. L., Broadhurst T., Kim K., Li T. G. F., Smoot G. F., 2019, arXiv e-prints, p. arXiv:1903.04513
Hannuksela O. A., Haris K., Ng K. K. Y., Kumar S., Mehta A. K., Keitel D., Li T. G. F., Ajith P., 2019, arXiv e-prints, p. arXiv:1901.02674

Haris K., Mehta A. K., Kumar S., Venumadhav T., Ajith P., 2018, arXiv e-prints, p. arXiv:1807.07062

Hongsheng Z., Xilong F., 2018, arXiv e-prints, arXiv:1809.06511

KAGRA Collaboration 2018, Progress of Theoretical and Experimental Physics, 2018

Lai K.-H., Hannuksela O. A., Herrera-Martín A., Diego J. M., Broadhurst T., Li T. G. F., 2018, Phys. Rev. D, 98, 083005

Hongsheng, Z., \& Xilong, F. 2018, arXiv:1809.06511

Lawrence J. K., 1971, Nuovo Cimento B Serie, 6, 225

Li S.-S., Mao S., Zhao Y., Lu Y., 2018, MNRAS, 476, 2220

Liao K., Biesiada M., Fan X.-L., 2019, arXiv e-prints, arXiv:1903.06612

Matsunaga N., Yamamoto K., 2006, Journal of Cosmology and Astro-Particle Physics, 2006, 23

Misner C. W., Thorne K. S., Wheeler J. A., 1973, Gravitation

Nakamura T. T., 1998, Phys. Rev. Lett., 80, 1138

Nakamura T. T., Deguchi S., 1999, Progress of Theoretical Physics Supplement, 133, 137

Ohanian H. C., 1974, International Journal of Theoretical Physics, 9, 425

Piórkowska, A., Biesiada, M., \& Zhu, Z.-H. 2013, J. Cosmology Astropart. Phys., 10, 022

Rosswog S., 2015, International Journal of Modern Physics D, 24, 1530012

Schneider P., Ehlers J., Falco E. E., 1992, Gravitational Lenses

Sereno M., Jetzer P., Sesana A., Volonteri M., 2011, Monthly Notices of the Royal Astronomical Society, 415, 2773

Sereno M., Sesana A., Bleuler A., Jetzer P., Volonteri M., Begelman M. C., 2010, Phys. Rev. Lett., 105, 251101

Smith G. P., Jauzac M., Veitch J., Farr W. M., Massey R., Richard J., 2018, MNRAS, 475, 3823

Takahashi R., 2017, The Astrophysical Journal, 835, 103

Takahashi R., Nakamura T., 2003, The Astrophysical Journal, 595, 1039

The LIGO Scientific Collaboration the Virgo Collaboration 2018, arXiv e-prints, p. arXiv:1811.12907

Varvella M. A., Angonin M. C., Tourrenc P., 2004, General Relativity and Gravitation, 36, 983

Wei J.-J., Wu X.-F., 2017, Monthly Notices of the Royal Astronomical Society, 472, 2906

Wood A., Mao S., 2005, MNRAS, 362, 945 